\title{
Effect of supraspinatus deficiency on humerus translation and glenohumeral contact force during abduction
}

\author{
Alexandre Terrier ${ }^{\mathrm{a}, *}$, Adrian Reist ${ }^{\mathrm{a}}$, Arne Vogel ${ }^{\mathrm{a}}$, Alain Farron ${ }^{\mathrm{b}}$ \\ a Laboratoire de Biomécanique en Orthopédie EPFL-HOSR, STI-IGBM-LBO, Bâtiment AA.BO, Station 15, \\ Ecole Polytechnique Fédérale de Lausanne (EPFL), 1015 Lausanne, Switzerland \\ ${ }^{\mathrm{b}}$ Hôpital Orthopédique de la Suisse Romande, Université de Lausanne, Lausanne, Switzerland
}

Received 28 September 2006; accepted 26 January 2007

\begin{abstract}
Background. Supraspinatus deficiency is the most frequent and important problem associated to rotator cuff pathologies. It reduces shoulder stability and can lead to osteoarthritis. The goal of this study was to develop a numerical model of the shoulder to analyse the biomechanical consequences of this pathology.

Methods. A 3D finite element model of the shoulder was developed from a normal cadaver specimen. It included the scapula, the humerus and the major abduction muscles. Instead of the usual ball-socket assumption, which prevents the natural translation of the humerus, shoulder stability was actively achieved by muscles. A feedback algorithm was developed to synchronise muscle forces during abduction. The numerical algorithm was validated against an algebraic model, and the calculated muscle moment arms were compared to the literature. Two cases were considered: a normal shoulder and the same one without supraspinatus.

Findings. For the normal shoulder, the model predicted the initial upward migration of the humeral head. The maximal humerus translation occurred at $30^{\circ}$ of abduction and was $0.75 \mathrm{~mm}$ above its ideal centered position. Without supraspinatus, it was 1.6 times higher and the contact point in the glenoid fossa was more eccentric. For the normal shoulder, the maximal glenohumeral force was $81 \%$ of the body weight, at $82^{\circ}$ of abduction. Without supraspinatus, it increased by $8 \%$, while the increase of muscle forces was $30 \%$.

Interpretation. Supraspinatus deficiency increased the upward migration of the humerus, the eccentric loading, and the joint and muscle forces, which may cause a limitation of active abduction and degenerative glenohumeral changes (osteoarthritis and the rotator cuff tear). (c) 2007 Elsevier Ltd. All rights reserved.
\end{abstract}

Keywords: Shoulder; Glenohumeral joint; Rotator cuff tear

\section{Introduction}

The rotator cuff tear is one of the most frequent pathologies of the shoulder, especially for aging individuals. This disease can affect all muscles of the rotator cuff, but the supraspinatus is almost always involved, and the tear is often limited to it. Rotator cuff tear is clinically associated to pain and functional disability, and also to higher osteoarthritis occurrence (Hsu et al., 2003). From a biomechanical point of view, the main consequence of a rotator cuff

\footnotetext{
* Corresponding author.

E-mail address: alexandre.terrier@epfl.ch (A. Terrier).
}

tear is a loss of stability, which results in a higher upward migration of the humeral head during abduction, and possible impingement with the acromion (Kaneko et al., 1995; Weiner and Macnab, 1970).

Several cadaver models have already been used to analyse the loss of shoulder stability in relation to a rotator cuff tear. Using a dynamic shoulder testing apparatus, active scapular abduction was simulated to study the effect of massive rotator cuff tears (Konrad et al., 2006). For a normal shoulder, there was only a slight upward translation of humeral head, while with massive rotator cuff tears, the upward translation was much more important, and could even lead to subacromial impingement. In another cadaver study, repaired supraspinatus was compared to 
pathologic and simulated complete tear of the supraspinatus (Yu et al., 2005). A significant increase of contact pressure was observed between repaired and simulated complete supraspinatus tear, but only inferior translation of the humeral head was observed. The anterior and posterior stability were also measured before and after tearing of the rotator cuff on cadaver shoulders (Hsu et al., 1997). Applying predefined muscle forces on 10 cadaver shoulders, the effect of a resection of the supraspinatus, associated with a resection of the infraspinatus, was analysed more specifically (Mura et al., 2003). After a supraspinatus resection, the average increase of upward migration of the humeral head was about $1 \mathrm{~mm}$, and it reached almost $3 \mathrm{~mm}$ without the infraspinatus. This study also showed that there is an important inter-individual variation (after supraspinatus resection, translation ranged from 0 to $5 \mathrm{~mm}$ ). The effect of a progressive tear of the rotator cuff on the glenohumeral joint force during abduction was also evaluated (Parsons et al., 2002). No significant differences was found after a complete tear of the supraspinatus, however, extension of tears beyond the supraspinatus tendon into the anterior and posterior aspect of the rotator cuff led to a significant decrease in the magnitude of joint reaction force. In another simulated abduction on cadavers, the supraspinatus deficiency required a higher force in the middle deltoid (101\%) to initiate abduction, but only $12 \%$ at full abduction (Thompson et al., 1996). No significant alterations in humeral translation were observed in that study.

Although all above cadaver studies confirmed a loss of stability of the glenohumeral joint after a partial or massive rotator cuff tear, some measurements and analyses could be improved with numerical models. However, despite the importance of this pathology, there are still no numerical models that can predict the natural translation of the humerus during abduction, which is a key factor for shoulder stability. This lack is probably caused by the relative biomechanical complexity of the joint. Indeed, conversely to the knee or the hip, which are mainly passively stabilized (ligaments or congruency), the shoulder is mainly actively stabilized by specific contractions of the surrounding muscles, which provides its wide range of motion.

Therefore, the first objective of this paper was to develop a 3D finite element model of the shoulder, using muscle stabilization, rather than the usual ball-socket assumption. The second objective was to analyse the effect of a supraspinatus deficiency on the humerus translation and the glenohumeral contact force, during continuous active abduction in the scapular plane. The position of the contact point on the glenoid surface and the muscle forces were also analysed.

\section{Methods}

The present finite element model was built from the same anatomical data as another shoulder model previously developed in our laboratory (Buchler et al., 2002). Computed tomography scans of a normal cadaver shoulder were used to reconstruct the geometry of the scapula and humerus, and dissection of the same shoulder provided muscle origins and insertions. Six muscles were considered here: middle deltoid (MD), anterior deltoid (AD), posterior deltoid (PD), supraspinatus (SS), subscapularis (SC), and infraspinatus combined with teres minor (IS). The model was then scaled so as to set the radius of the humeral head to $24 \mathrm{~mm}$, for future comparison purpose. The articular cartilage was reconstructed from general anatomical observations: an elliptical surface was fitted at the bony side (McPherson et al., 1997), while the articular side was represented by a spherical surface with a radius of $26 \mathrm{~mm}$, which was positioned in such a way that the thickness in the middle was $2 \mathrm{~mm}$ (Soslowsky et al., 1992a). Muscles were modelled partly by $3 \mathrm{D}$ volumes and by cables (Fig. 1).

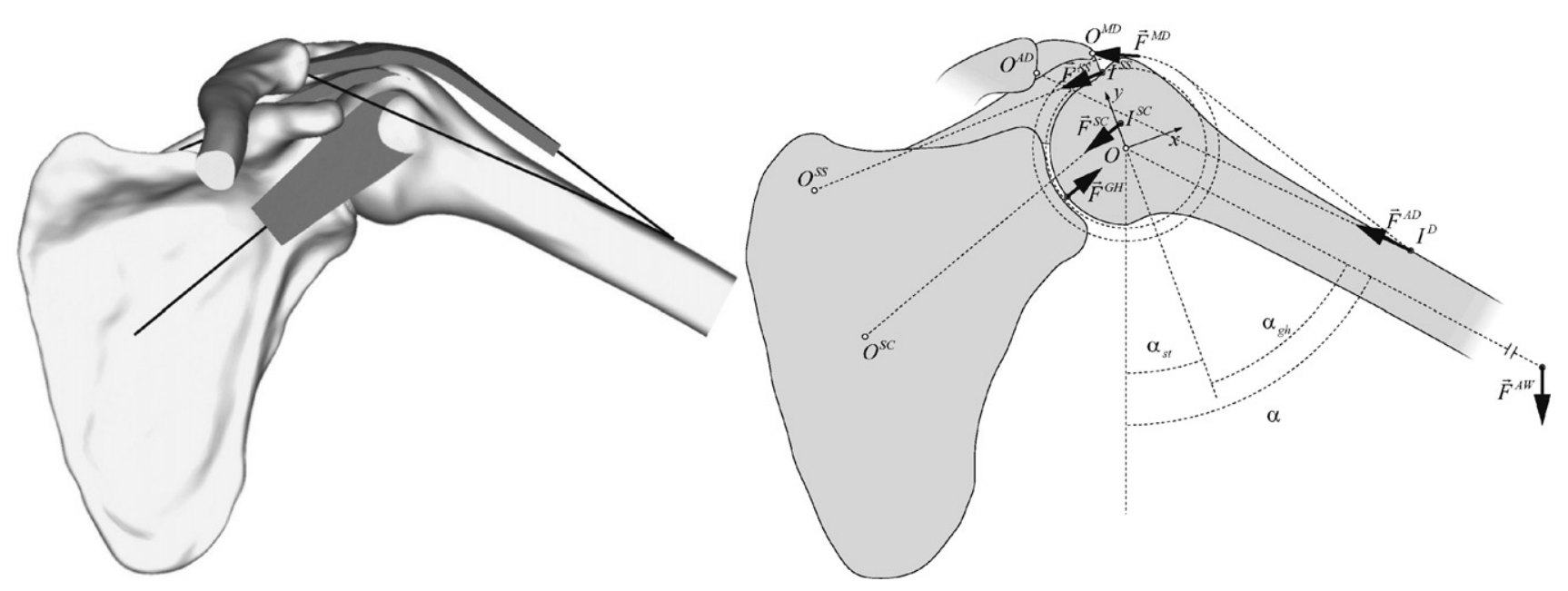

Fig. 1. 3D model (left) and the corresponding simplified 2D algebraic model (right). On the 3D model, rotator cuff muscles and MD are solid deformable bodies that wrap around the humeral head to stabilize the joint. In the 2D model MD and SS also wrap around the humeral head, but the stability is guaranteed by the ball-socket assumption. 
Even with only six muscles, there were more unknown forces than available equilibrium equations. This indeterminate in the muscular forces was characterized by five ratios, relating each muscle force to the MD force:

$r^{\mathrm{MD}}=\frac{F^{\mathrm{MD}}}{F^{\mathrm{MD}}} \equiv 1, \quad r^{\mathrm{AD}}=\frac{F^{\mathrm{AD}}}{F^{\mathrm{MD}}}, \quad r^{\mathrm{PD}}=\frac{F^{\mathrm{PD}}}{F^{\mathrm{MD}}}$,

$r^{\mathrm{SC}}=\frac{F^{\mathrm{SS}}}{F^{\mathrm{MD}}}, \quad r^{\mathrm{IS}}=\frac{F^{\mathrm{IS}}}{F^{\mathrm{MD}}}, \quad r^{\mathrm{SS}}=\frac{F^{\mathrm{SC}}}{F^{\mathrm{MD}}}$

To numerically constrain these ratios to a predefined value, a user-defined element (UEL) was developed and implemented within the finite element software Abaqus (Abaqus, Inc.). This UEL synchronized the muscles through a feedback mechanism, where the shortening of MD controlled the muscle forces and the elevation of the arm. As for standard deformable finite elements, the UEL was called at each increment of the Newton-Raphson algorithm. Therefore, at each increment, both mechanical equilibrium equations and ratios relations were fulfilled. The UEL had six pairs of nodes: one for each muscle. The first two nodes were used as a force captor for the MD, while the other five pairs were used to set the amplitude and direction of the other muscles. The contribution of the UEL to the Jacobian operator (tangent stiffness of the Newton-Raphson algorithm) matrix is

$K^{\mathrm{MN}}=-\frac{\mathrm{d} F^{\mathrm{M}}}{\mathrm{d} u^{\mathrm{N}}}$

where $F^{\mathrm{M}}$ are the contributions of the UEL to the residual vector force, and $u^{\mathrm{N}}$ are the nodal displacements of the UEL. Each $F^{\mathrm{M}}$ is a function of $u^{1}$ to $u^{6}$, the three orthogonal displacements of the first two nodes used for the MD force captor. Except for MD, each $F^{\mathrm{M}}$ additionally depends on the six displacements of the two points that define the orientation of the muscle associated to $F^{\mathrm{M}}$. An exact algebraic expression for all forces $F^{\mathrm{M}}$ and matrix elements $K^{\mathrm{MN}}$ was obtained and implemented in the UEL.

Bones were rigid, while cartilage and muscles were deformable. Cartilage was characterized by a Neo-Hookean potential $w=1.8\left(I_{1}-3\right)$, where $I_{1}$ is the first invariant of the Cauchy-Green tensor (Buchler and Farron, 2004). The muscle anisotropy was modelled with parallel stiff fibres embedded along the principal direction of the muscle volume, which was described by a soft Neo-Hookean potential $w=0.5\left(I_{1}-3\right)$. Linear hexahedral elements were used for cartilage and solid muscles, while truss elements (no bending stiffness) were used for embedded fibres. The same truss elements were also used for the cable part of the muscles (Fig. 1). The wrapping of the muscles on the humerus was achieved through the interaction between the contact surfaces.

Abduction was simulated in the scapular plane and in neutral rotation. The ratios of the muscle forces were determined from the product between electromyography (EMG) and physiological cross-sectional area (PCSA), as initially proposed by Poppen and Walker (1978). EMG and PSCA were estimated from the literature (Ringelberg, 1985; John-
Table 1

The muscles ratios were estimated by the product between PCSA and EMG, which were obtained from the literature (Ringelberg, 1985; Johnson et al., 1996; Kronberg et al., 1990), and normalized to the MD values

\begin{tabular}{lllllll}
\hline & MD & AD & PD & SS & SC & IS \\
\hline PCSA & 1.0 & 1.0 & 1.0 & 0.5 & 1.5 & 1.5 \\
EMG & 1.0 & 0.8 & 0.2 & 1.0 & 0.3 & 0.3 \\
Ratios & 1.0 & 0.8 & 0.2 & 0.5 & 0.5 & 0.5 \\
\hline
\end{tabular}

son et al., 1996; Kronberg et al., 1990). Since EMG are nearly proportional during scapular abduction, the ratios were assumed constant in this case (Table 1), solving this way the indeterminate. The arm weight was set to $37.5 \mathrm{~N}$ ( $5 \%$ of the body weight) and applied at the arm gravity center, $32 \mathrm{~cm}$ from the humeral head center (Poppen and Walker, 1978). To reproduce the correct orientation of the arm weight force, the scapula was continuously rotated during arm elevation, according to the scapulohumeral rhythm, which was set to 2:1 (e.g. Poppen and Walker, 1976).

This model was validated in two steps. First, the numerical accuracy of the UEL was assessed by restricting the numerical model in such a way as to correspond to a simplified algebraic 2D model (illustrated in Fig. 1 and fully described in Supplementary data). The difference in the glenohumeral force of the simplified numerical model and the algebraic one was a measure of the numerical error. Second, the biomechanical coherence of the model was confirmed by comparing the moment arms of the muscles to cadaver measurements reported in the literature.

To evaluate the effect of a SS deficiency, the SS was completely removed and the following variables were compared to those of the normal shoulder: inferior-superior translation of the humerus, glenohumeral contact force, position of the contact area center on the glenoid surface and muscle forces.

\section{Results}

The comparison of the simplified numerical model with the algebraic model produced a relative error in the glenohumeral contact force that was below 1\% (Fig. 2). The difference between the glenohumeral contact force obtained with the complete 3D model (Fig. 5) and the algebraic one was less than 3\%. The moment arms curves (Fig. 3) confirmed that MD is the main abductor muscle. During abduction, AD increased its moment arm, as well as PD, the latter being antagonist below $50^{\circ}$ of glenohumeral abduction. Conversely, SS was more efficient at lower angles of abduction. SC and IS moment arms were smaller and also decreasing during abduction.

The translation of the humeral head was measured relatively to the glenoid, in the inferior-superior direction. Zero translation corresponded to a perfect centering of the humeral head in the glenoid fossa (Fig. 4). In the initial neutral position, the humeral head was in equilibrium on 


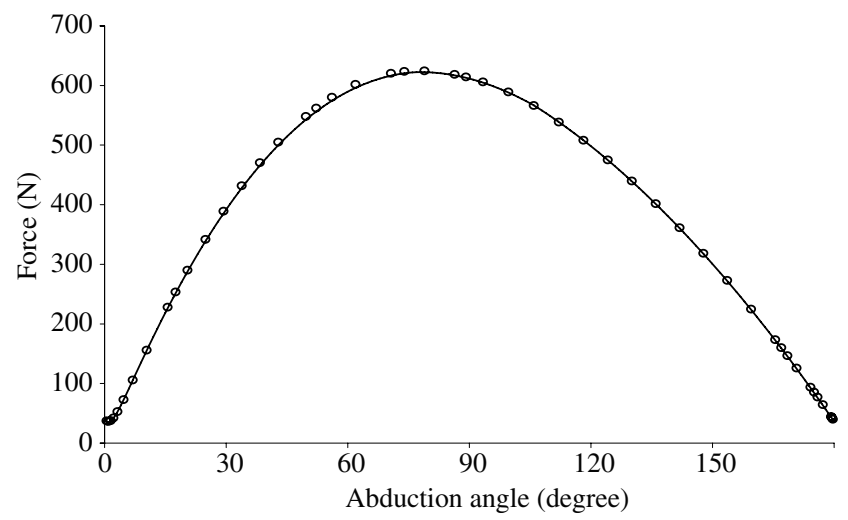

Fig. 2. Glenohumeral contact force obtained from the exact algebraic solution of the simplified model (continuous curve) compared to the numerical solution of the simplified model (circles). The relative error is below $1 \%$.

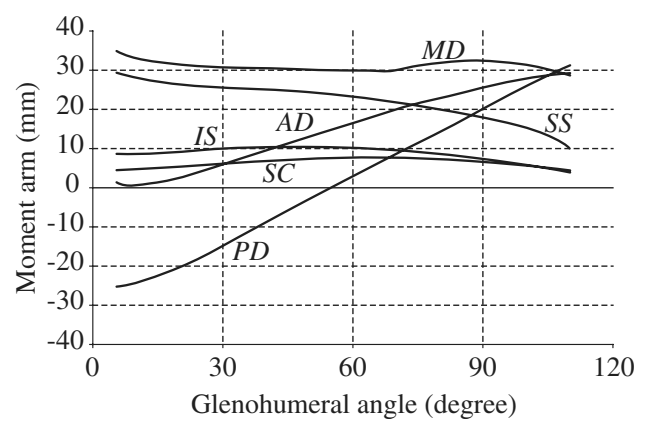

Fig. 3. Moment arms of the muscles during abduction in the scapular plane, as a function of the glenohumeral angle. The dominant role of MD is confirmed. SS becomes less efficient during abduction, while AD and PD become more efficient. PD is antagonist below $50^{\circ}$ of glenohumeral angle, which corresponds to $75^{\circ}$ of abduction angle.

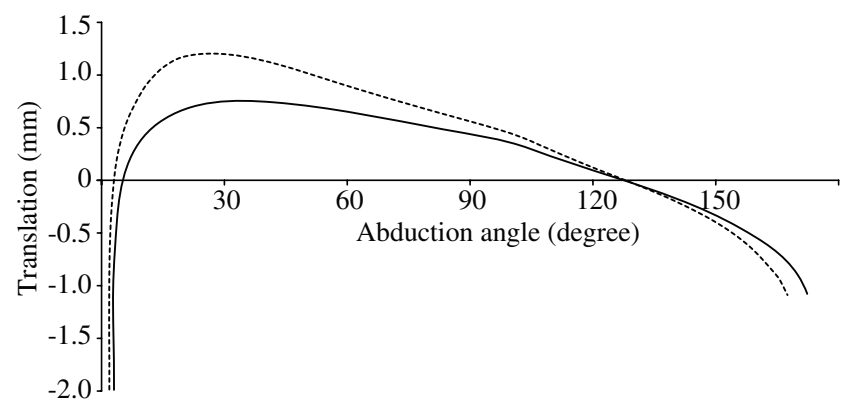

Fig. 4. Inferior-superior translation of the humeral head relatively to the glenoid during scapular abduction, for the normal shoulder (continuous line) and without SS (dotted line). In the vertical axis, zero corresponds to a perfect centering of the humeral head in the glenoid fossa.

the inferior border of the glenoid (Fig. 6A). During the first $30^{\circ}$ of abduction, the humeral head migrated superiorly by about $3 \mathrm{~mm}$, but was only $0.75 \mathrm{~mm}$ above its ideal center. From $30^{\circ}$ of abduction, there was an almost constant downward translation, with a perfect centering of the humeral head near $130^{\circ}$ of abduction. At $150^{\circ}$ of abduction the humerus head was only $0.5 \mathrm{~mm}$ below the ideal center posi-

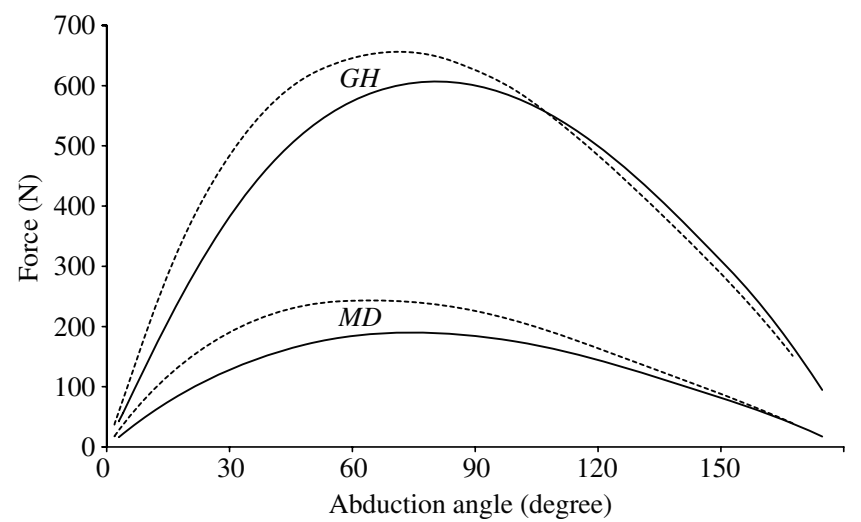

Fig. 5. Glenohumeral $(\mathrm{GH})$ contact force and $\mathrm{MD}$ force, for the normal shoulder (continuous line) and without SS (dotted line), during shoulder abduction. For the normal shoulder the maximal GH force occurred at $82^{\circ}$ of abduction and corresponded to $81 \%$ of the body weight. Without $\mathrm{SS}$, the maximal GH force increased by $8 \%$, while the maximal DM force increased by $30 \%$.

tion. Without SS, the translation of the humeral head followed the same trend, but the maximal upward migration above the ideal center was 1.6 times higher and reached $1.2 \mathrm{~mm}$.

For the normal shoulder, the glenohumeral force was maximal at $82^{\circ}$ of abduction and corresponded to $81 \%$ of the body weight $(608 \mathrm{~N})$. The MD force was maximal at $75^{\circ}$ of abduction and reached $25 \%$ of the body weight $(190 \mathrm{~N})$. The other muscle forces were proportional to the MD force, according to the muscle ratios. Without SS, glenohumeral and muscle forces followed the same trend, but the maximum glenohumeral force was $8 \%$ higher, while the maximum muscle forces were $30 \%$ higher (Fig. 5). Note also that these maxima occurred earlier (at 70 , respectively, $64^{\circ}$ of abduction).

As it might be expected, the inferior-superior position of the contact area on the glenoid surface was correlated to the translation of the humeral head (Fig. 6). For the normal shoulder, the contact was initially located on the inferior rim of the glenoid (Fig. 6A) and moved on the superior part of the glenoid during the first $30^{\circ}$ of abduction. It slowly returned to the inferior part during the rest of the abduction, being in the center of the glenoid near $130^{\circ}$ of abduction (Fig. 6D). Without SS, the center of the contact area on the glenoid surface was more eccentric, particularly during the initial upward migration of the humeral head.

\section{Discussion}

Although the rotator cuff tear is a common pathology of the shoulder after 60 years of age, it is still not well understood. The goal of the present study was first to develop a 3D numerical model of the shoulder and second to analyse the effect of a rupture of the supraspinatus tendon on the translation of the humerus and the forces in the glenohumeral joint. The numerical part of the model was validated against a simplified algebraic model, while the biomechan- 


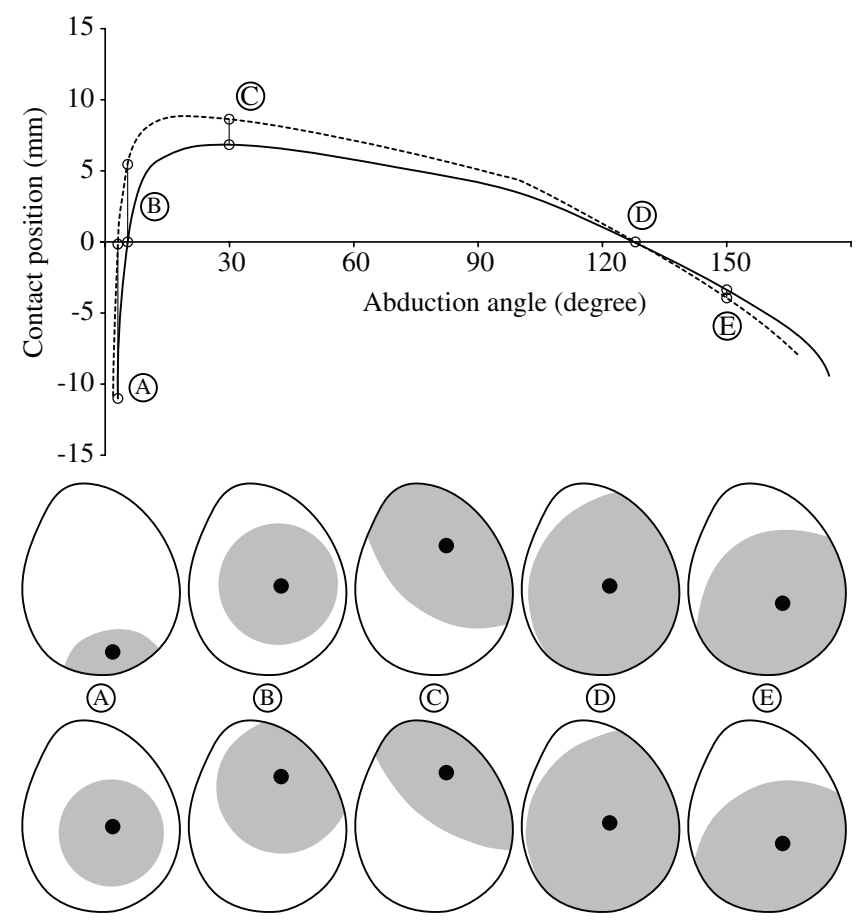

Fig. 6. The two curves represent the inferior-superior position of the contact point on the glenoid, for the normal shoulder (continuous line) and without SS (dotted line). In the vertical axis, zero corresponds to the center of the glenoid, and the lower and upper limits to the inferior and superior edge of the glenoid surface. Below is the corresponding contact area at five typical positions (A-E), for the normal shoulder (first line) and without SS (second line). The contact area (gray zone) corresponds to nonzero contact pressure, and contact point (black dot) correspond to the application point of the equivalent contact force.

ical part was validated against published measurements of the muscle moment arms. The results of this model confirmed that a SS deficiency has an important effect on the natural translation of the humeral head, but also on the muscle and joint forces during scapular abduction.

The comparison of the numerical solution to the algebraic one, for the simplified model, proved that the numerical model, and in particular the UEL subroutine, could reproduce the exact solution of the mechanical system with a sufficient accuracy. For the shoulder, as for any joint, it is clear that moment arms of the muscles are crucial in the biomechanics of the joint. Moment arms were measured using the standard excursion method, for each muscle, during the entire abduction. The resulting curves corresponded well with published cadaver measurements (e.g. Liu et al., 1997; Otis et al., 1994). Although the humerus was free to translate in any direction, only the translation in the inferior-superior direction was presented here. The media-lateral translation was obviously constrained by the glenoid surface, and the amplitude of the translation in the anterior-posterior direction was small (less than $0.5 \mathrm{~mm}$ ) during the entire range of abduction. After its initial upward migration, the humeral head remained rather centered in the glenoid fossa during the entire movement. The increase in the initial upward migration caused by the resection of the SS tendon, clearly demonstrated the loss of stability that may occur after a tearing of this tendon. The effect of the SS deficiency on the glenohumeral contact force was not as obvious as its effect on the muscle forces. It seems indeed rational that the removal of the SS muscle, which is an important abductor muscle, regarding to its moment arm, should be compensated by the others muscles. However, the increase of the glenohumeral force caused by the SS deficiency is not so intuitive and can only be explained through the equilibrium equations; the same effect was predicted with the simplified algebraic model.

Even if limited to abduction, the biomechanical analysis of the shoulder remains difficult, not only because of the central role of the muscles in the stabilization of the joint, but mainly due to the mathematical indeterminate of this mechanical system. In the literature, the indeterminate problem has been solved by four different methods: by avoiding it altogether by using only one muscle (Inman et al., 1944), by using EMG measurements (Poppen and Walker, 1978), by using optimization methods (van der Helm, 1994; Karlsson and Peterson, 1992; Favre et al., 2005), and by using constant force ratios (Wuelker et al., 1995; Apreleva et al., 2000) or other predetermined muscle forces (Soslowsky et al., 1992b). As for the above cadaver models, the constant ratios method used in the present paper seems quite reasonable for abduction in the scapular plane, since all abductor muscles are nearly symmetrical in this plane and produce nearly proportional EMG signals (Ringelberg, 1985). Most biomechanical models simplify the muscular stabilization mechanism by modelling the glenohumeral joint as a ball-socket joint (Poppen and Walker, 1978; van der Helm, 1994; Karlsson and Peterson, 1992). Although this hypothesis is quite reasonable for a force analysis (only 3\% difference of maximal joint force when ball-socket was used in the model), the joint stability can only be estimated by the projection of the reaction force on the glenoid surface, rather than with the humeral head translation. The glenohumeral force obtained here was very close to the classical $80-90 \%$ of the body weight reported by Poppen and Walker (1978). Using the cadaver model of Soslowsky et al. (1992b), Kelkar et al. (2001) measured the natural translations of the humerus during scapular abduction. The general trend and amplitude of translation predicted by the present numerical model were very close to these experimental measurements. Using open-MRI, Graichen et al. (2000) obtained also similar results, which confirms the initial observations of Poppen and Walker (1976). The effect of a progressive cuff deficiency was investigated by Konrad et al. (2006), using the cadaver model of Debski et al. (1995). After a complete SS tear and $50 \%$ tear of SC and IS, they also observed an increase of the initial upward migration. Similar results were obtained on cadavers by Mura et al. (2003), who measured $0.8 \mathrm{~mm}$ of average increase in initial upward migration after SS resection. The predicted change of force in MD after SS deficiency also corresponded fairly well with the cadaver study of Sharkey et al. (1994), where a 
progressive deactivation of the rotator cuff muscle was simulated. Indeed, after SS deactivation, the maximum MD force occurred about $15^{\circ}$ earlier and was about $10 \%$ higher, compared to $11^{\circ}$ and $30 \%$ of force increase predicted by the model.

As for most of the cadaver studies, this numerical analysis was limited to abduction since it is the most important movement of the shoulder: mechanically, because it induces large glenohumeral forces, and functionally, since it is essential for most activities of daily living. More precisely, abduction was performed in the scapular plane, which can justify the simplifying hypothesis of constant muscle ratios during the entire abduction. Indeed, EMG signals measured in those abductor muscles were almost proportional during abduction in that plane (Ringelberg, 1985). Moreover, when the elevation is performed in the plane of the scapula, the muscles are almost symmetric about that plane.

The original feature of the present model is its ability to account for the stability of the glenohumeral joint through the muscles and the articular contact surfaces, instead of the usual ball-socket assumption. The main advantage of this method is to allow for the natural translation of the humeral head, which is crucial to analyse the stability of the glenohumeral joint. This model was used to analyse the effect of a deficiency of the supraspinatus, which can be caused by a rotator cuff tear. The resulting increase of humerus upward migration and muscle forces may partly explain the occurrence of osteoarthritis and progression of the tearing to the other tendons of the rotator cuff.

\section{Acknowledgments}

This study was partly supported by Tornier S.A.S. (Montbonnot, France). The authors specially thank Y.-A. Ratron (R\&D Tornier S.A.S) for his advises.

\section{Appendix A. Supplementary data}

Supplementary data associated with this article can be found, in the online version, at doi:10.1016/j.clinbiomech. 2007.01.015.

\section{References}

Apreleva, M., Parsons, I.M., Warner, J.J., Fu, F.H., Woo, S.L., 2000. Experimental investigation of reaction forces at the glenohumeral joint during active abduction. J. Shoulder Elbow Surg. 9, 409-417.

Buchler, P., Farron, A., 2004. Benefits of an anatomical reconstruction of the humeral head during shoulder arthroplasty: a finite element analysis. Clin. Biomech. 19, 16-23.

Buchler, P., Ramaniraka, N.A., Rakotomanana, L.R., Iannotti, J.P., Farron, A., 2002. A finite element model of the shoulder: application to the comparison of normal and osteoarthritic joints. Clin. Biomech. 17, 630-639.

Debski, R.E., McMahon, P.J., Thompson, W.O., Woo, S.L., Warner, J.J., Fu, F.H., 1995. A new dynamic testing apparatus to study glenohumeral joint motion. J. Biomech. 28, 869-874.
Favre, P., Sheikh, R., Fucentese, S.F., Jacob, H.A., 2005. An algorithm for estimation of shoulder muscle forces for clinical use. Clin. Biomech. 20, 822-833.

Graichen, H., Stammberger, T., Bonel, H., Karl-Hans, E., Reiser, M., Eckstein, F., 2000. Glenohumeral translation during active and passive elevation of the shoulder - a 3D open-MRI study. J. Biomech. 33, 609613.

Hsu, H.C., Luo, Z.P., Cofield, R.H., An, K.N., 1997. Influence of rotator cuff tearing on glenohumeral stability. J. Shoulder Elbow Surg. 6, 413422.

Hsu, H.C., Luo, Z.P., Stone, J.J., Huang, T.H., An, K.N., 2003. Correlation between rotator cuff tear and glenohumeral degeneration. Acta Orthop. Scand. 74, 89-94.

Inman, V.T., Saunders, J.B., Abbott, L.C., 1944. Observations of the function of the shoulder joint. J. Bone Joint Surg. Am., 1-30.

Johnson, G.R., Spalding, D., Nowitzke, A., Bogduk, N., 1996. Modelling the muscles of the scapula morphometric and coordinate data and functional implications. J. Biomech. 29, 1039-1051.

Kaneko, K., De Mouy, E.H., Brunet, M.E., 1995. Massive rotator cuff tears. Screening by routine radiographs. Clin. Imaging 19, 8-11.

Karlsson, D., Peterson, B., 1992. Towards a model for force predictions in the human shoulder. J. Biomech. 25, 189-199.

Kelkar, R., Wang, V.M., Flatow, E.L., Newton, P.M., Ateshian, G.A., Bigliani, L.U., Pawluk, R.J., Mow, V.C., 2001. Glenohumeral mechanics: a study of articular geometry, contact, and kinematics. J. Shoulder Elbow Surg. 10, 73-84.

Konrad, G.G., Markmiller, M., Jolly, J.T., Ruter, A.E., Sudkamp, N.P., McMahon, P.J., Debski, R.E., 2006. Decreasing glenoid inclination improves function in shoulders with simulated massive rotator cuff tears. Clin. Biomech. 21, 942-949.

Kronberg, M., Nemeth, G., Brostrom, L.A., 1990. Muscle activity and coordination in the normal shoulder. An electromyographic study. Clin. Orthop. Relat. Res., 76-85.

Liu, J., Hughes, R.E., Smutz, W.P., Niebur, G., Nan-An, K., 1997. Roles of deltoid and rotator cuff muscles in shoulder elevation. Clin. Biomech. 12, 32-38.

McPherson, E.J., Friedman, R.J., An, Y.H., Chokesi, R., Dooley, R.L., 1997. Anthropometric study of normal glenohumeral relationships. J. Shoulder Elbow Surg. 6, 105-112.

Mura, N., O'Driscoll, S.W., Zobitz, M.E., Heers, G., Jenkyn, T.R., Chou, S.M., Halder, A.M., An, K.N., 2003. The effect of infraspinatus disruption on glenohumeral torque and superior migration of the humeral head: a biomechanical study. J. Shoulder Elbow Surg. 12, $179-184$.

Otis, J.C., Jiang, C.C., Wickiewicz, T.L., Peterson, M.G., Warren, R.F., Santner, T.J., 1994. Changes in the moment arms of the rotator cuff and deltoid muscles with abduction and rotation. J. Bone Joint Surg. Am. 76, 667-676.

Parsons, I.M., Apreleva, M., Fu, F.H., Woo, S.L., 2002. The effect of rotator cuff tears on reaction forces at the glenohumeral joint. $\mathrm{J}$. Orthop. Res. 20, 439-446.

Poppen, N.K., Walker, P.S., 1976. Normal and abnormal motion of the shoulder. J. Bone Joint Surg. Am. 58, 195-201.

Poppen, N.K., Walker, P.S., 1978. Forces at the glenohumeral joint in abduction. Clin. Orthop., 165-170.

Ringelberg, J.A., 1985. EMG and force production of some human shoulder muscles during isometric abduction. J. Biomech. 18, 939947.

Sharkey, N.A., Marder, R.A., Hanson, P.B., 1994. The entire rotator cuff contributes to elevation of the arm. J. Orthop. Res. 12, 699-708.

Soslowsky, L.J., Flatow, E.L., Bigliani, L.U., Mow, V.C., 1992a. Articular geometry of the glenohumeral joint. Clin. Orthop., 181-190.

Soslowsky, L.J., Flatow, E.L., Bigliani, L.U., Pawluk, R.J., Ateshian, G.A., Mow, V.C., 1992b. Quantitation of in situ contact areas at the glenohumeral joint: a biomechanical study. J. Orthop. Res. 10, 524 534.

Thompson, W.O., Debski, R.E., Boardman 3rd, N.D., Taskiran, E., Warner, J.J., Fu, F.H., Woo, S.L., 1996. A biomechanical analysis of 
rotator cuff deficiency in a cadaveric model. Am. J. Sports Med. 24, 286-292.

van der Helm, F.C., 1994. Analysis of the kinematic and dynamic behavior of the shoulder mechanism. J. Biomech. 27, 527-550.

Weiner, D.S., Macnab, I., 1970. Superior migration of the humeral head. A radiological aid in the diagnosis of tears of the rotator cuff. J. Bone Joint Surg. Br. 52, 524-527.
Wuelker, N., Wirth, C.J., Plitz, W., Roetman, B., 1995. A dynamic shoulder model: reliability testing and muscle force study. J. Biomech. 28, 489-499.

Yu, J., McGarry, M.H., Lee, Y.S., Duong, L.V., Lee, T.Q., 2005. Biomechanical effects of supraspinatus repair on the glenohumeral joint. J. Shoulder Elbow Surg. 14, 65S-71S. 